\title{
Water Recycling and Filtration
}

\author{
Anna Pocquette, Nick Angelino, Sam Allegretti, Kyle Reusch, Alya Abd Aziz, and \\ Bala Maheswaran \\ College of Engineering \\ Northeastern University
}

\begin{abstract}
Access to usable and clean water is arguable the most valuable resource on Earth. The objective of our final project is to be able to recycle and filter greywater on site, in an affordable and portable way. Our mission is to be able to produce this system for people in rural and impoverished areas that do not have access to an abundance of clean water. This machine will be able to provide a space on top to clean or work with whatever the consumer needs (ie. washing clothes, dishes, etc.). From there, the contaminated water will be filtered through many methods that will be explained later, and deposited into a clean reservoir that the consumer will be able to access immediately to reuse this water. We added a unique pressure system to the filter piece to efficiently drive the contaminated water through, making our device more fastacting and versatile. We are striving to conserve the clean water, making resources more attainable to more people, as well as making these resources last longer.
\end{abstract}

Our filter holds a lot of potential for consumer use. The inexpensive design, portability, and effectiveness of our product make it a viable aid to developing nations in need of water infrastructure. The speed of our innovative pressured filtration system makes our product a favorable substitute for other sand filters. The largest problem future and current generations will face is the effects of climate change.

\section{Introduction:}

If we can improve water supply and sanitation can boost a developing country's economy, but more importantly can greatly reduce poverty and deadly diseases. Contaminated water is linked to a multitude of diseases as stated previously, most commonly cholera, diarrhea, dysentery, Hepatitis A, typhoid, and polio. When wastewater from urban, industrial, and agricultural uses are inadequately managed, the water sources of hundreds of millions of people are dangerously contaminated or chemically polluted. In addition to water's health effects, socioeconomic effects are also felt worldwide. When water comes from improved and more accessible sources, people spend less physical effort and time attaining it, meaning they can be productive in different ways. This also results in an increase in overall personal safety because they don't take as risky of journeys to collect water, which may transfer diseases if not from a reliable source. With children at an even higher risk of water-borne illnesses, access to better and more efficient water sources can improve school attendance, which overall shows positive effects on society as a whole. According to World Health Organization, climate change, increasing water scarcity, population growth, demographic changes and urbanization already pose challenges for water supply systems. By 2025, half of the world's population will be living in water-stressed areas. Re-use of wastewater, to recover water, nutrients, or energy, is becoming an 
important strategy. Increasingly countries are using wastewater for irrigation - in developing countries this represents $7 \%$ of irrigated land. While this practice if done inappropriately poses health risks, safe management of wastewater can yield multiple benefits, including increased food production. In 2010, the UN General Assembly explicitly recognized the human right to water and sanitation. Everyone has the right to sufficient, continuous, safe, acceptable, physically accessible, and affordable water for personal and domestic use. This brings us to our proposed solution: a portable, affordable and durable water recycling system that you can wash clothes and dishes in and reuse this wastewater for similar, daily tasks. We created a water filtration system with a basin on top, which makes washing dishes and clothes easy and efficient, a valve which allows the water to stay in the basin until you want to filter it, a pressure system that drives water the the filter at a quick pace, a filter piece that actually filters the water for recycling, and a collection basin at the bottom. Early on, we outlined a multitude of goals to follow throughout our design process and experimentation. Our general goals include portability, simplicity, and for the device itself to be inexpensive to manufacture and repair. Our more specific goals will be described in greater detail within the design portion. Since water is such an important resource, we were able to pull together many studies to aid us in prototyping and design.

\section{Applicable Studies and Information:}

From the beginning of the brainstorming process, we knew we wanted to deal with grey wastewater and integrated water recycling, rather than just focusing on a simple water filter. The most feasible option seemed to be a gravel-sand filter, which will be outlined later. There was one particular study that we drew inspiration from: a case study put out by the Australian Government regarding wastewater and how to recycle it. Greywater is wastewater from non-toilet plumbing fixtures such as showers, basins and taps. Greywater is ideal for garden watering, with the appropriate precautions, such as using low or no sodium and phosphorus products and applying the water below the surface. Appropriately treated greywater can also be reused indoors for toilet flushing and clothes washing, both significant water consumers. There are many advantages to recycling this greywater. For example, use fewer water resources, the ability to irrigate the garden during drought or water restrictions and cutting down the amount of pollution going into waterways. Greywater can be reused in gardens with little or no treatment. Treated greywater can be reused for toilet flushing and clothes washing, two of a household's biggest users of water. Reusing treated greywater for toilet flushing can save approximately 50L of water in an average household every day. Reusing treated greywater in a clothes washer can save approximately 90L of water in an average household every day. Treatments include coarse filtration to remove large particles, including hair, and to prevent clogging. It can be as simple as a waterproof box and a filter bag or stocking attached with rubber bands. Another treatment is fine filtration and biological treatment, using a sand filter and reed bed 
combination. Microbes in the sand break down organic matter in the water and the reeds take up nutrients. The basic structure is a waterproof box filled with coarse sand laid over a gravel bed.

\section{Methods}

The goal of our design was to make a water recycling system that will filter waste water from dish washing. The basic design that we wanted to accomplish was a way to collect the waste water from washing, a filter for that water, and a way to collect the water that has been filtered. More specific design goals included a pressure system to push the water through the filter faster, a sand filter as the filter system, and general portability.

Our design process started with the pressure system of the filter and the housing of the filter. Our vision was to have a tube with a sand filter that took in dirty water at the top and cleaned the water of contaminants to output at the bottom. The pressure system would push the water through the sand filter faster than gravity would, thus classifying the entire system as a rapid sand filter as opposed to a slow sand filter. We came up with multiple variations of pressure systems. Our designs included a piston that would push water through and a lever that Figure 1: Drawing of Piston Design would push water through. We ultimately landed on the idea to use air pressure from a pump to push the water through, a design that was considered a backup for the initial ideas.

After determining which design we would use, we started our building by making a PVC tube holding the sand filter. We clamped a piece of cheese-cloth to the bottom of the
PVC pipe and started filling the pipe with our filtration materials. We first filled the tube about $25 \%$ with activated charcoal, then a layer of cheese-cloth. Next we filtered the large particles out of the sand we had bought using a strainer, and filled the PVC about $25 \%$ with the fine sand that we strained, added a layer of chees-cloth, and then filled the tube with about $20 \%$ of the rough, unstrained sand. Finally we added another layer of cheese-cloth, then filled about $10 \%$ of the tube with gravel that we washed off in a sink to get rid of the small particles. We left headspace in the tube to allow for more room for water to collect above the filter so we can push more water through at once.

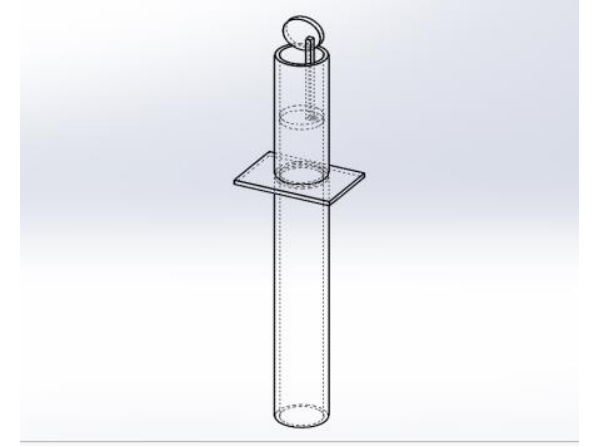

Figure 1: Drawing of Piston Design

After making the system that would be filtering water, the next step was to build around this filter to make it a usable product. We added functional PVC parts to the top of the sand filter PVC to make a pressure system. We connected a T-joint to the filter tube and connected a bike valve to the perpendicular opening. We cut the valve piece off a bike tire with a little extra rubber, and attached that piece to the T-joint PVC part with a ring clamp. Then we attached a rotating valve to the top of the T-joint, to be able to open and close the system to the 
outside, thus allowing for pressure to be built inside.

With the pressure system for the filter completed, we had to build a way to collect the water and feed the water into the turnable valve so it can be pushed through the filter. To do this we $3 \mathrm{D}$ printed a tube piece that fit onto the PVC coming out of the top of the valve and glued it into a hole that we drilled into a bucket that would be collecting the water. We made the 3D printed part using the 3D printers at Northeastern University's First Year Engineering Learning and Innovation Center (FYELIC). We put a drain filter over the hole in the bucket that leads into the PVC, and sealed the connection with plumber's putty.

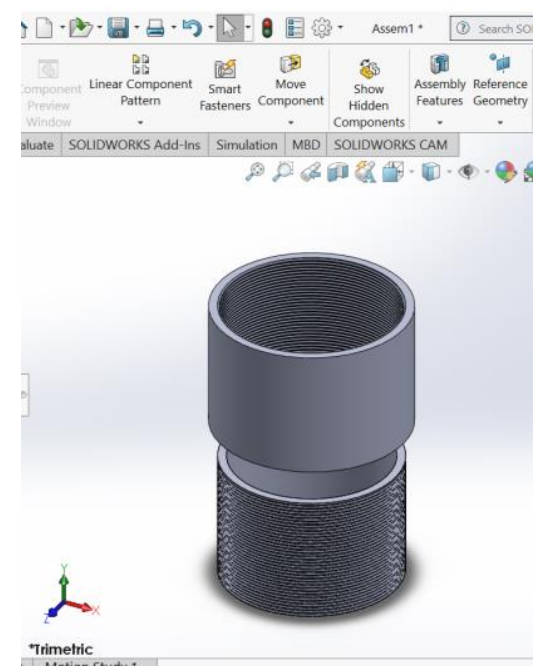

Figure 2: 3D Printed Part in SolidWorks

With the functional aspect of the water filter completed, a structural component of the filter was needed, as the filter could not stand up on its own. We purchased four $\operatorname{lin} x \operatorname{lin} x$ $3 \mathrm{ft}$ wooden shafts, and acquired a thin board of plywood from a wood scrap pile in FYELIC. First, we cut a hole in the middle of the plywood board so that it would hold up the PVC tubing; the hole was designed to rest on the lip between the turnable valve and the PVC pipe under it. We cut the wooden shafts to equal lengths with a hand saw, and drilled each of them to the four corners of the thin plywood board. The height of the wooden shafts was made long enough so that it would hold up the water filter above the ground, so there could be a bucket placed under the filter to collect water. Then the excess wooden shaft that was cut off was used to prop up the top water collecting bucket; we cut the wooden shafts with a handsaw so that they would allow for the bucket to rest on them, and then drilled the shafts into the plywood so that they would line up with the bucket.

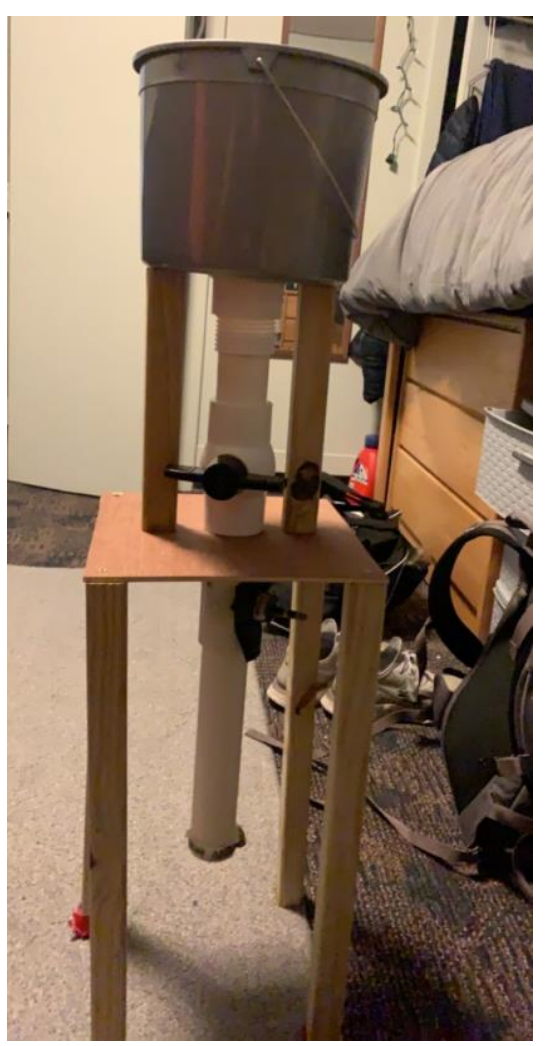

Figure 3: Water Filter Final Product

\section{Design: Details and Practicality}

In short, the water filter follows a simple design with upright tubing and a wooden 
stand. Water is introduced into the filter through a basin at the top. This functions as a place to soak and wash clothes, and can be filled with water to allow for washing dishes. The basin connects to the 3D printed screw using hot glue and plumber's putty. Having the basin screw into the rest of the filter allows it to be easily disconnected to replace and clean the filter.

Next, there is a PVC valve separating the filter from the basin. This allows for a closed system in which pressure can be applied, pushing the water through the filter. Our filter was designed with an inner diameter of $1.5 \mathrm{in}$ to satisfy the pressure recommendations for a fast sand filter of 14 psi. Below the valve is a T-joint which uses a bike tire and corresponding pump to create pressure for the filter. A metal clamp is used to make an airtight seal between the valve and the PVC filter. Finally, the filter is located at the bottom of the device. It consists of gravel and sand, decreasing in coarseness as the water drains. Throughout the filter layers of cheesecloth are used, and at the very bottom there is a layer of activated charcoal. This was used because the fine porous structure of activated carbon is able to trap volatile organic compounds (VOCs), chlorine, and other sediments.

To support the filter, there is a wooden stand that the filter rests on. A 2 inch hole was drilled through a piece of wood, corresponding to the outside diameter of the PVC. The filter runs through this piece, and is supported with 4 legs. 2 pieces of wood are connected to the top of this table, supporting the basin.

\section{Results and Discussion}

Our initial goal was to effectively filter and recycle greywater for future use with washing dishes and clothes. When dirty water is run through the filter, there is a visible increase in water quality. With polluted water, the depth at which the secchi disk is visible increased after running the water through the filter, which leads us to believe that the filter is able to trap dirt and other particulates effectively.

Using the results from our $\mathrm{pH}$ testing, we can confidently say that the filter removed detergents from the water. Lightly soapy water had a visible drop in $\mathrm{pH}$, going from 7.8 to a 7.2. If the filter were perfectly efficient at removing these chemicals, the $\mathrm{pH}$ would be 7.0, however this is not the case. Since the purpose of the recycled greywater is to be used for washing clothes and dishes, this aspect of the design is still successful. If the dirt in the water is filtered, with some soap left behind, this will cut down on the amount of soap used by a consumer. This makes the filter more cost effective, as conserves resources used for washing materials.

While our design is portable, it is a bit bulky and hard to move. The wooden stand is functional, but not extremely elegant when it comes to supporting the filter. One future improvement would be a designated handle or strap on the side of the product. This would increase the portability of the filter.

The current product is a good starting point for what could be a very reliable water recycling system. Our future plans would be 
to make more improvements so that system is practical, cost effective, and effective for our goals. Currently the arrangement of the valves means there is a low capacity and the system is complicated. A new value system that allows for a tighter seal and increased capacity would be ideal. Also, the design does not have a reservoir for the water that is pressurized so it takes a while between uses so addition volume would help solve this problem. An improvement that would make the filter disease resistant and help prevent water borne illnesses is a UV light system, since these illnesses are a major problem in areas that do not have access to clean water. Streamlined materials that could be sourced cheaper and easier that would better fit our goal like custom pump or pipes would also be added and a redesign of the housing and the shape of the filter to increase portability. These improvements would be part of a new design to possibly be marketed

\section{Data}

The water filter is designed to clean the water by removing the large particulates in the water by passing it through finer and finer mediums to remove particles and to neutralize the water by running it through activated charcoal. Testing the water filter consisted of two primary methods: a secchi disk to test for clarity and particulates and a $\mathrm{pH}$ test kit to determine the alkalinity of the water through the filter.

The secchi disk is a device that measures the clarity of water. It consists of a large black and white disk that is lowered into the water and the depth that the disk is no longer visible is recorded and compared to a baseline to determine the clarity and therefore how clean the water is overall. We adapted this for our project by using a button that was about 3 inches in diameter at the bottom of a clear water bottle with an open top. We compared the water and dirt mixture before being filtered and after and measured the depth that the disk was not visible. This gave us a numerical value to compare the clarity of the water along with the initial visual observations of the water. What we found for the dirty and soapy water was that the depth was 2.6 inches before filtering and 7.4 inches after filtering. For the water and dirt mixture the initial visibility was 2.625 inches then 8 inches after filtering and visually much clearer after the second round of filtering, which did not have enough depth in the bottle to test.

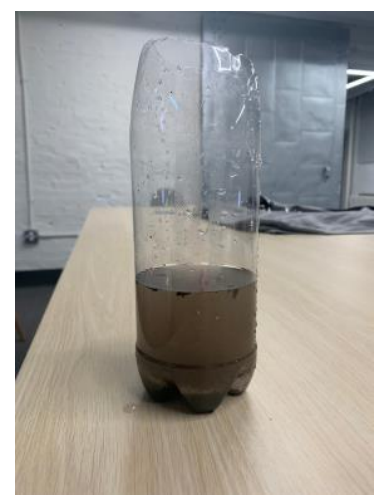

Figure 4: Dirt and Water before filtering

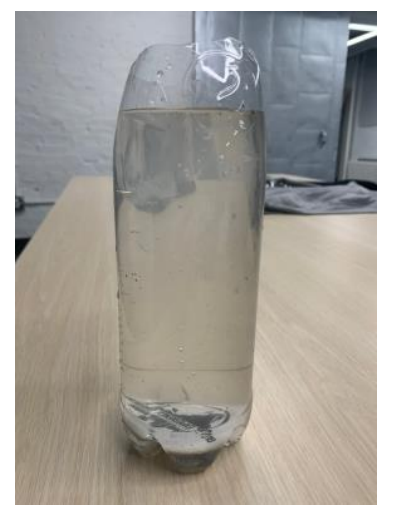

Figure 5: Dirt and Water after filtering 


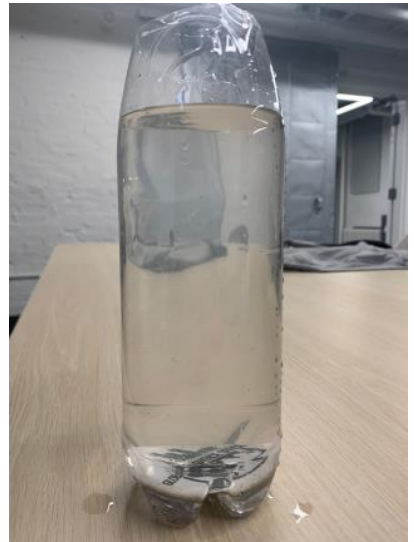

Figure 5: Dirt and Water after filtering twice

The $\mathrm{pH}$ testing was done to see the alkalinity of the water after filtering as washing dishes or clothes adds soap to the water which raises the $\mathrm{pH}$ of the water to basic levels that are not potable. We purchased a $\mathrm{pH}$ testing kit that used a powder that changed color based on the $\mathrm{pH}$ of the water, with blue being very basic, green being neutral and red being very acidic. We test some of the soap water before putting it through the filter and determined that water had a $\mathrm{pH}$ of about 7.8 and a $\mathrm{pH}$ of 7.2 after filtering, meaning that it was successful in lowering the $\mathrm{pH}$ of the water to a lower level.

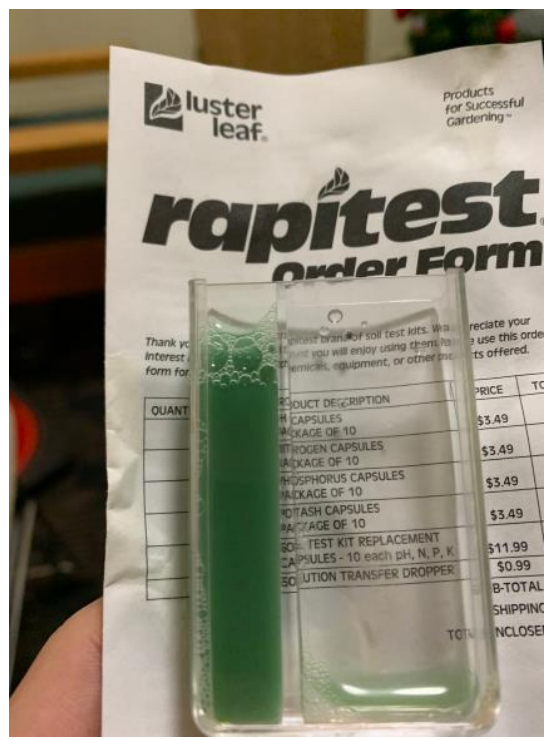

Figure 7: pH Testing Solution of UnFiltered Water

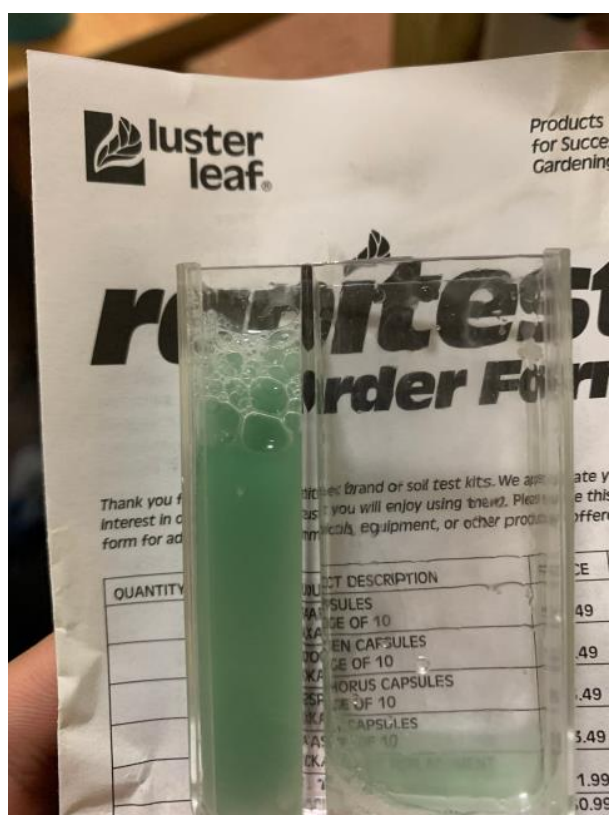

Figure 8: $\mathrm{pH}$ Testing Solution of Filtered

Water

\section{Data Analysis}

The results of our testing show that our filter was successful in accomplishing the tasks we wanted the filter to do. The water was noticeably cleaner and the speed of the filter is much faster than a drip filter. We ran the filter through without using the pump and it took over an hour so the pump was very effective in making the filter practical. Further testing would look at different ranges of $\mathrm{pH}$ and different water conditions to see the extent to which the filter would be effective. Another addition would be testing different ratios of materials in the filter to see what combination was most effective for the cost. Further designs could include field testing kits that would ensure that the water being filtered would be properly filtered and that it was safe to drink. 
It is crucial that our product is extensively tested before its implementation in a public setting as the effects of a nonfunctioning product could be devastating. Proper education and instruction on the use of the filter are also important in maintaining an ethical product so that the consumer is informed on its function and limitations. Sourcing of the materials should also be examined and made sustainable. The filter is intended to be a solution to environmental and economic problems and the production of our water filter should mirror that with sustainable production. The price of the product should also be low since the communities it is designed for do not have high economic output and cannot afford to pay for a product that is too high in cost. Distribution of the filter is also important. Reaching remote areas with our filter will be challenging and efforts should be made to ensure that the filter reaches as many people as possible.

\section{Conclusion}

Our filter holds a lot of potential for consumer use. The inexpensive design, portability, and effectiveness of our product make it a viable aid to developing nations in need of water infrastructure. The speed of our innovative pressured filtration system makes our product a favorable substitute for other sand filters. The largest problem future and current generations will face is the effects of climate change. Droughts will increase in frequency and intensity, with developing nations being the most devastated. The importance of water recycling will only increase, and we hope our product will be able to catalyze the growth and ease of water recycling. We believe our design ideas can further be improved and implemented to meet these needs. We also believe that future improvements could extend the product's effectiveness to be able to output drinking water. Our filter is one step towards eliminating all waterborne disease deaths in developing countries; if improvements are made, production and distribution of our fitler would have a large benefit on the health of developing countries. We hope to see a large push by engineers worldwide to look for solutions for issues that developing countries are facing.

\section{References}

1. Recover wastewater resources locally, Zhiyong Jason Ren \& Art K. Umble, Nature 529, 25(2016)

2. Membraneless water filtration using CO2, Sangwoo Shin, Orest Shardt, Patrick B. Warren, and Howard A. Stone, Nat. Commun. 8, 15181 (2017)

3. Fane, Simon, and Chris Reardon. "Wastewater Reuse." Edited by Geoff Milne, YourHome, Australian Government, 2013, www.yourhome.gov.au/water/wastewate r-reuse.

4. "Drinking-Water." World Health Organization, World Health Organization, www.who.int/newsroom/fact-sheets/detail/drinking-water. 